\title{
MITTEILUNGEN
}

\section{Hohenheimer Magnesium-Symposium}

24./25. September 1982

Tagungsort:

Themen:

Veranstalter:

Auskunft/Anmeldung:
Stuttgart-Hohenheim (Universität)

Magnesium

Chemie, Boden, Pflanzen, Tierernährung

Zufuhr über Lebensmittel

Physiologie, Pathophysiologie, Klinik

Herz/Kreislauf Experimentelle Studien

Gesellschaft für Magnesium-Forschung e. V.

Dr. Joachim Helbig

Schriftführer

Postfach 1256

8132 Tutzing, Telefon (0 81 58) 6071 und 6072

Für die Schriftleitung verantwortlich: Prof. Dr. K. H. Bässler, Physiologisch-Chemisches Institut, Universität Mainz, Saarstraße 21, 6500 Mainz, und Prof. Dr. A. Fricker, Bundesforschungsanstalt für Ernährung, Engesserstraße 20, 7500 Karlsruhe 1 - Anzeigenverwaltung: Springer-Verlag GmbH \& Co. KG, Kurfürstendamm 237, D-1000 Berlin 15 - Verantwortlich für den Anzeigenteil: E. Lückermann Verlag: Dr. Dietrich Steinkopff Verlag, Postfach 11 10 08, Saalbaustraße 12, 6100 Darmstadt 11 - Satz und Druck: Ungeheuer + Ulmer KG GmbH + Co, Körnerstraße 14-18, 7140 Ludwigsburg. 